\title{
The Effect of Visual Stimulus Characteristics on Adolescents' Short-Term Memory
}

\author{
Yixuan $\operatorname{Jin}^{1, *, \dagger}$, Yingying $\operatorname{Liu}^{2, *, \dagger}$
}

\author{
${ }^{1}$ Ulink Collage Shanghai, Shanghai, China \\ ${ }^{2}$ The Godolphin School, Hangzhou, China \\ *Corresponding author. Email: ' yixuan.jin@ulink.cn \\ ${ }^{t}$ These authors contributed equally.
}

\begin{abstract}
In the past, many studies suggested the importance of short-term memory and memory associated with age, but not many researches have specifically targeted to the short-term memory related to teenagers. Therefore, this research will focus on testing whether the use of visual stimulus in learning process can have a positive impact on short-term memory for adolescents. There are19 participants involved in the experiment (9 males), aging from 15-18. The experiment is a laboratory experiment, which used repeated measure design. The independent variable (IV) in this experiment is the colour of the visual stimulus used, and the IV consists 3 experimental conditions, including words with solid colour, coloured picture, and black and white picture. The results obtained show that coloured picture has the highest correction rate, whilst black and white condition has smallest mean respond time and standard deviation. To some extent, this paper reveals the influence of colour characteristics of visual stimuli on short-term memory. Further studies could be made based on the importance of visual stimulus, especially colour and images.
\end{abstract}

Keywords: Short-term memory, teenagers, visual stimulus, learning, recognition

\section{INTRODUCTION}

Memory has been defined as a broadest sense, that includes Learning, Retention, Association and Reproduction [1]. Atkinson-Shiffrin memory model has been investigated on the structure of the memory, where the model has suggested there are three stages in the memory system, including sensory memory, short-term memory and long-term memory respectively [2]. The information will enter the sensory memory system at first, then due to the process of encoding, such information will turn to short-term memory. After the process of consolidation, the information will then turn to long-term memory, where people need to retrieve the memories in the brain from a long time ago. Short-term memory is a component of memory, also known as active memory or primary memory, which is the ability for the brain to store, but not manipulating, a relatively small amount of information in a short time period. Also, according to the study conducted by Miller, it suggested for short-term memory, there are some limitations involved, including the amount of information and the duration for the information can be stored in each period [3].
The reason to focus on investigating the short-term memory is because that the short-term memory is the first step in the memory process, and it is prior to the long-term memory. In addition, experiment published in 2014 which carried out by Yana Fandakova et al. [4] suggested that better item-context binding was associated with higher working memory performance, which means that developmental differences in binding mechanisms are closely related to working memory development in childhood and old age. Therefore, it is essential to carrying more research on the short-term memory on teenage years and the possible ways to improve the short-term capacity for teenagers.

Different characteristics on visual stimulus can have different impacts on the short-term memory for instance, using different levels of emotional intensity stimulus. The study of Canli et al. [5] investigated on whether the emotive images will be remembered better than those that have a little emotional impact on an individual, and the study has suggested that the more emotionally intense an image is, the more likely it will be remembered. Another study carried out by Liu in 1997 examined the effect of different types of visual stimulus on short-term memory, and the study used 6 
types of visual stimulus including Arabic numerals, English letters, meaningful and meaningless pictures and Chinese characters with meaning and non-sense [6].

Nevertheless, whether the normal-rating pictures that do not have the high emotional intensity and arousal level can or cannot enhance the short-term memory have not been examined in the past studies. Also, whether the effects of using visual aids, like colourful pictures or diagrams in education can enhance memory or they are just simply a source of distraction for the student is still unknown. Furthermore, the past studies have deeply investigated in the forms of words or characters given in the learning process, but have not been investigate very deeply on the use of colour. Therefore, this study is aiming to investigate on two areas: firstly, can the use of visual stimuli, like pictures in the learning process, enhance our short-term memory. Second aim is to test whether the colour plays an important role in the process of remembering and thus enhance human memory. There are two one-tailed hypotheses drawn: 1) The use of pictures will improve short-term memory. 2) The unexpected memory recall scores on the coloured-picture condition (condition 1) will be higher than the black-and-white picture condition (condition 2). The study adopts repeated measure design and conducted in a quiet and standardized classroom. There are in total 19 participants involved by opportunity sampling.

\section{METHOD}

\subsection{Participants}

The subjects in the experiment were 19 healthy, right-handed teenagers, including 9 males and 10 females, with the age ranging from 15-18 $(\mathrm{M}=16.7$, SD $=0.93)$. They were recruited by the opportunity sampling in the Ulink collage Shanghai.

\subsection{Design and Apparatus}

The experiment used a repeated measure design, which means all the participants undergone the exact same procedure but with the different order of the visual stimulus. There is one independent variable (IV) in the experiment with 3 treatments, including one control group: solid color group (Figure $1 \mathrm{a}$ ), two experimental groups which are colored picture group (condition 1, figure $1 \mathrm{~b}$ ), and black and white picture group (condition 2, figure $1 \mathrm{c}$ ).

The dependent variable in the study is the percentage of incorrect answers and mean response time that participants get under each condition. The only apparatus used in the experiment is the laptop and the application for the design of the experiment is PsychoPy.

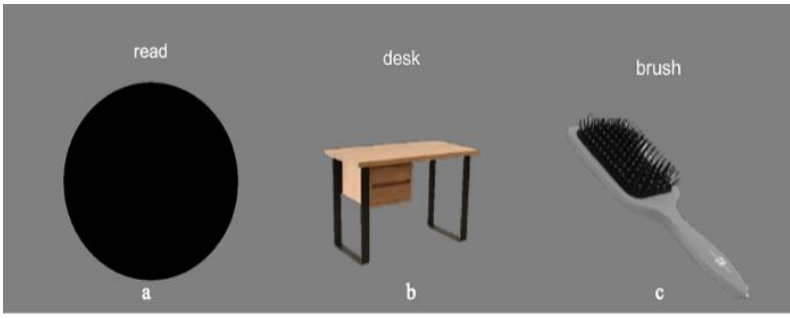

Figure 1. Picture used in each treatment

Notes: A, controlled condition; B: colored-picture condition; C: black and white picture condition

\subsection{Procedure}

The experimenter will ask for the agreement on the participation in the experiment from the subjects before they had been taken to a quiet room by the experimenter. Then the experimenter will give instruction to the participants using standardized prods "please type down your nickname and your gender, you can write any name you want, for example like 'chair'." If the participants asked whether they can type the opposite gender, the second prods will be followed "No you cannot, but it will still provide enough confidentiality." After the name and gender had been collected the experiment will be started.

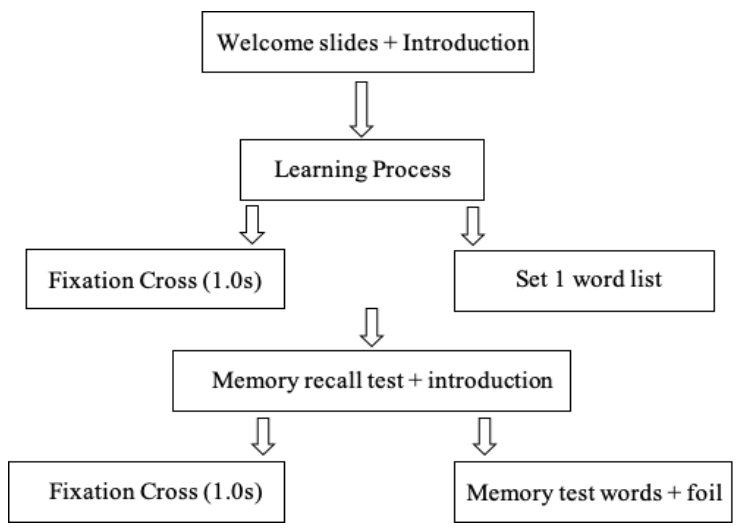

Figure 2. Experiment procedure in PsychoPy

In the introduction, the participants will be given informed consent, where the false aim will be given to reduce the possibility for subjects to guess the real aim of the study, thus reducing demand characteristic. Confidentiality and protection will be also mentioned in the informed consent. Moreover, the participants will be asked again if they are sure they want to continue the experiment by given the right to choose whether to click the mouse to continue the test or not.

In the learning task, in a total of 18 words will be shown, and have been divided into three condition groups. Each slide of words will be shown for 1 second and followed by 1 second of the fixation cross. The size (letter height 0.05$)$ and position $(0,0.18)$ of the fixation cross will be exactly the same as the one for the words 
throughout the study, and the font used was Arial with the color white.

Table 1. Choice of words in Set 1 wordlist used in learning process

\begin{tabular}{|c|c|c|c|}
\hline & 4 letters & 5 letters & 6 letters \\
\hline $\begin{array}{l}\text { Black and } \\
\text { white }\end{array}$ & I & $\begin{array}{l}\text { Banana, Ruler, } \\
\text { Brush }\end{array}$ & $\begin{array}{l}\text { Create, Rubber, } \\
\text { Orange }\end{array}$ \\
\hline $\begin{array}{l}\text { Colored } \\
\text { picture }\end{array}$ & $\begin{array}{l}\text { Wash, Desk, } \\
\text { ear }\end{array}$ & Apple, Drink & Pencil \\
\hline Solid color & $\begin{array}{l}\text { Read, Kiwi, } \\
\text { case }\end{array}$ & Paper, Grape & Forget \\
\hline
\end{tabular}

In the memory recall test, subjects need to press the keyboard to answer the questions on whether they saw the words in the learning task. Press " $y$ " for yes, " $n$ " for no, and "m" for not sure. In the study, the participants will be given 19 words where 9 of them had existed in the learning task ( 3 words under each condition), and 10 foils been selected with the same category of the words used in the learning task. The use of foils in the experiment aims to ensure that the participants paying attention to the words in the learning task.

Table 2. Memory test words lists used in memory recall test

\begin{tabular}{ccc}
\hline Category & Words & $\begin{array}{c}\text { Correct } \\
\text { Answer }\end{array}$ \\
\hline $\begin{array}{c}\text { Color } \\
\text { Black and } \\
\text { White }\end{array}$ & $\begin{array}{c}\text { Apple, Pencil, Drink, } \\
\text { Create, Banana, Ruler }\end{array}$ & Y \\
$\begin{array}{c}\text { Solid Color } \\
\text { Foil }\end{array}$ & Read, Grape, Case & Y \\
& $\begin{array}{c}\text { Bear, Robert, Water, Ballpen, Peach, } \\
\text { Gun, Mallon, Mallet, Think, Speak }\end{array}$ & $\mathrm{N}$ \\
\hline
\end{tabular}

In the end, the debriefing will be taken apart, the appreciation will be made towards the subjects on the contribution and the true aim will be stated. Also, the experimenter will be at the side to answer every question raised by the participants.

\section{RESULTS}

Two set of results from this experiment have been obtained.

\subsection{Corrections}

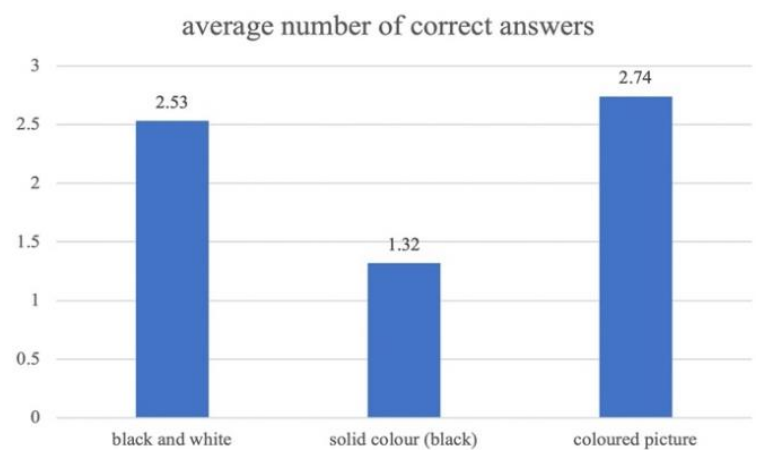

Figure 3. average number of correct answers in each condition
Note: 19 participants each were given 18 learning and 19 recognition vocabularies. Within 19 recognition vocabs, each condition had 3 target vocabs, remaining 10 vocabs in total were foil. In other words, average correct answers obtained out of 3 tasks. Hence the maximum correction number possible is 3 .

According to figure 3 , it could be seen that solid colour condition has the biggest effect on memorising, as well as being the condition with lowest correct answers (1.32 tasks answered correctly out of 3 tasks). Two pictured groups had similar results, with coloured picture group having slightly higher number of correct answers per participant per condition (2.74) than black and white condition (B\&W) (2.53).

\subsection{Mean respond time}

Second set of result is the mean respond time. In other word, the average time taken for the participants to respond and answer.

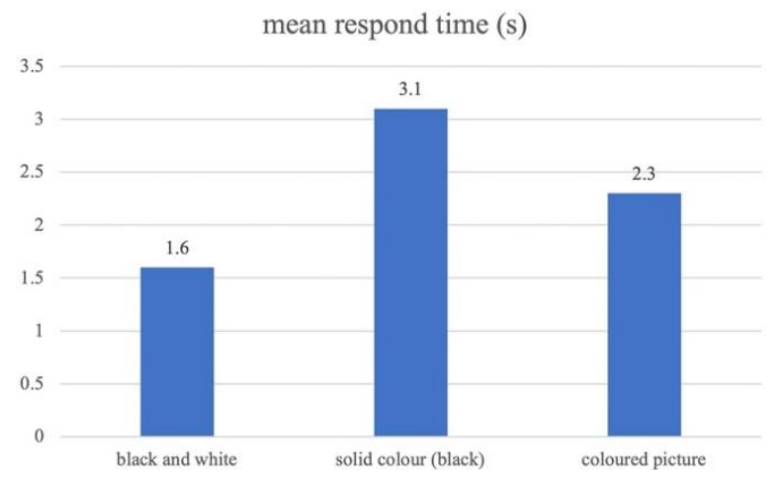

Figure 4. mean respond time (seconds)

In figure 4, it could be seen that solid colour condition had the highest respond time (RT), which was roughly 3 seconds and double the RT of B\&W condition. Coloured picture had a value of RT of 2.3 seconds.

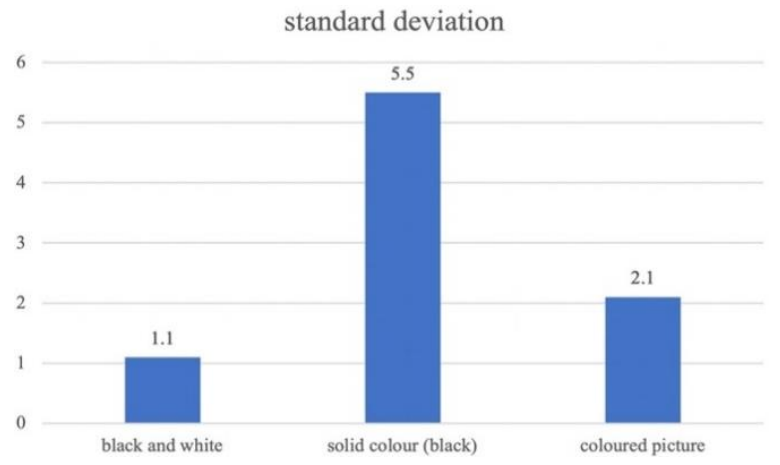

Figure 5. standard deviation (SD) of the respond times

Figure 5 presents the SD of the respond time in each condition. Similar number of correct answers, solid colour group had the highest spread of dada (5.5), followed by coloured picture group (2.1), then the B\&W group (1.1). 


\section{DISCUSSION}

\subsection{Summary of The Research}

The research has been made to investigate the effect of: 1. the use of image, and 2. role of colour in short term memory (STM). Experiment with 3 conditions were designed and carried out on 19 participants to test. The result obtained showed the use of image and colour would both help with STM, with having images or not being a bigger factor.

\subsection{Comparison with Previously Research}

Two hypotheses obtained were 1. use of pictures will improve short-term memory, and 2. recall scores on the colored-picture condition will be higher than the blackand-white picture condition (in other words, colored picture helps STM better). According to the results from the experiment, both hypotheses were proven true.

Unfortunately, little literacy resources could be found on this topic. From the limited articles acquired from Color relations increase the capacity of visual short-term memory by Sanocki \& Sulman [7], it could be seen that the use of color could indeed make a difference in memory, specifically, the use of similar-color would aid the distinguish between different colors, but not similar colors. The results between of $\mathrm{B} \& \mathrm{~W}$ and colored pictures would agree with the first part of this conclusion, for there is a difference between the results. However, the second part was unable to be justified, as their aim and topic are still loosely related to our aim. It is still premature for a reliable, scientific result to be made.

\subsection{Limitations, Improvements \& Future Direction}

The research and experiment in this paper had several upsides. First of all, the experiment has been done very precisely. Large scale of tasks was given to the participants, in both learning and testing phases. Secondly, extraneous variables are kept to a minimum, and for example, age, experiment situations and education levels were similar for the participants. Thirdly, being a lab experiment, standard procedural was used, and a causal relationship could be concluded. Lastly, ethics issue was handles delicately. Inform content and debriefing were provided to the participants. One slide of each was used, at the beginning and ending respectively. By doing so, participants were informed on the potential harm on the experiment, and the mental harm could be kept to the minimum.

However, drawbacks do exist. As one of the biggest flaws, the sample group was quite limited, only 19 participants were involved. This could lead to a major problem which is the lack of representativity. In other words, the result may not be suitable to be applied to the whole population. As a lab experiment, it is also low on ecological validity due to the fact that the set conditions and tasks were artificial and man-made on purpose, they are unlikely to happen in normal everyday life. This means that the result could only be used as a reference and cannot be applied to daily memorizing tasks. Lastly, despite the best effort put in, ethical issues, especially deception, could not be eradicated. This is mainly due to the intentional avoiding in demand characteristics.

For future researches, analysis could be made on the cause of differences, the effect of gender and how the use of visual stimulus could improve memory process.

\section{CONCLUSION}

According to the results, the hypothesis was matched. The use of pictures in learning can enhance people's shortterm memory significantly than the use of the texts only.

Based on our research and results, following conclusions could be made: solid color (black) had the biggest effect on memorizing, whether on correction or responding. On average, black and white pictures seem to help the memorizing the best, for it had concentrated and relatively short time for response, and a high value of correct recalling. The presence of color is trivial. The pictured groups had similar results in all the data.

\section{REFERENCES}

[1] Ebbinghaus, H. (1964). Memory/ A contribution to experimental psychology (H. A. Ruger, C. E. Bussenius Trans.). New York/ Dover. (Original work published 1885)

[2] Atkinson, R. C. (1968). Human memory: a proposed system and its control processes. Psychology of Learning \& Motivation, 2.

[3] Miller, G. A. (1956). The Magical number seven, plus or minus two: some limits on our capacity for processing information. Psychological Review, 63 (2), 81-97

[4] Fandakova, Y., Sander, M. C., Werkle-Bergner, M., \& Shing, Y. L. (2014). Age differences in short-term memory binding are related to working memory performance across the lifespan. Psychology \& Aging, 29(1), 140-149.

[5] Canli, T., Zhao, Z., Brewer, J., Gabrieli, J. D., \& Cahill, L. (2000). Event-related activation in the human amygdala associates with later memory for individual emotional experience. Journal of Neuroscience, 20(19), 99-0.

[6] Liu Xufeng, Huang-Fu En, Wang Xiaoying, Chen Zuhai (1997). Short term memory of various stimulus attributes. J Fourth Mili Med Univ 1997: 18(5)

[7] Sanocki, T., \& Sulman, N. (2011). Color relations increase the capacity of visual short-term memory. Perception, 40(6), 635. 